\title{
Role of matrix metalloproteinase-9 in angiogenesis caused by ocular infection with herpes simplex virus
}

\author{
Sujin Lee, Mei Zheng, Bumseok Kim, and Barry T. Rouse \\ Department of Microbiology, The University of Tennessee, Knoxville, Tennessee, USA
}

\begin{abstract}
In this report, we demonstrate that herpes simplex virus (HSV) infection of the cornea results in the upregulation of the matrix-degrading metalloproteinase enzyme MMP-9. This enzyme was shown to contribute to the neovascularization process that occurs in the corneal stroma in response to HSV infection. The likely source of MMP-9, at least initially after infection, was neutrophils that were signaled to invade the cornea soon after infection. Corneal infiltrating neutrophils were shown to express MMP-9, and preventing the neutrophil response with specific mAb diminished MMP-9 expression as well as the extent of angiogenesis. Further supporting a role for MMP-9 in HSV-induced corneal angiogenesis was the observation that inhibition of MMP-9 with the specific inhibitor TIMP-1 resulted in reduced angiogenesis. In addition, angiogenesis was diminished in ocularly infected MMP-9 knockout mice. Our results demonstrate that MMP-9 is involved in angiogenesis caused by HSV. Since angiogenesis appears to represent a vital step in the pathogenesis of herpetic stromal keratitis, these results indicate that targeting MMP-9 for inhibition should prove useful for the therapy of herpetic stromal keratitis.
\end{abstract}

J. Clin. Invest. 110:1105-1111 (2002). doi:10.1172/JCI200215755.

\section{Introduction}

One of the more distressing lesions caused by herpes simplex virus (HSV) infection is vision impairment and blindness resulting from a chronic immunoinflammatory reaction in the corneal stroma. Studies on experimental herpetic stromal keratitis (HSK) in animal models have revealed that the pathogenesis involves numerous cellular and molecular participants. One early essential event appears to be neovascularization of the normally avascular corneal stroma. This event is assumed to be necessary to assist corneal access of some of the cellular orchestrators of HSK (1). In line with this notion, inhibition of new blood vessel development serves to moderate the severity of HSK lesions (2). Currently, the mechanisms by which HSV infection results in corneal angiogenesis remain ill-defined, and more than likely multiple participants could be involved. These include the VEGF family of proteins, potent angiogenesis factors under both physiological and pathological circumstances and known to be produced following ocular infection with HSV (2). The VEGF proteins, as well as certain chemokines, induce angiogenesis by binding to receptors on vascular endothelial cells, causing them to undergo growth and

Received for publication April 22, 2002, and accepted in revised form August 20, 2002.

Address correspondence to: Barry T. Rouse, Department of Microbiology, M409 Walters Life Sciences Building, The University of Tennessee, Knoxville, Tennessee 37996-0845, USA. Phone: (865) 974-4026; Fax: (865) 974-4007; E-mail: btr@utk.edu. Conflict of interest: No conflict of interest has been declared. Nonstandard abbreviations used: herpes simplex virus (HSV); herpetic stromal keratitis (HSK); matrix metalloproteinase (MMP); ultraviolet (UV); Chinese hamster ovary (CHO); extracellular matrix (ECM). movement. Other molecules influence angiogenesis by breaking down the extracellular matrix, thus facilitating neovessel growth. Matrix-degrading proteases include the collagenases, for example, matrix metalloproteinase-2 (MMP-2) and MMP-9 and heparanases (3). Several MMP proteins appear involved in tumor angiogenesis, but the role of such molecules in viralinduced angiogenesis has not been described.

In the present report, we have evaluated if MMP-9 is involved in angiogenesis following infection of the mouse eye with HSV-1. Our results demonstrate that MMP-9, while undetectable in normal eyes, is produced in the cornea in response to HSV infection. A prominent cell type that produces MMP early after infection is invading neutrophils. When MMP-9 levels were suppressed (as could be achieved by neutrophil depletion) by inhibition with the specific inhibitor TIMP-1 or by using MMP-9 knockout mice, HSVinduced angiogenesis was inhibited. In addition, the results further demonstrate that inhibition of angiogenesis is reflected in the reduced severity of subsequent HSK lesions. Our observations are discussed in terms of the value of controlling angiogenesis in the therapy of herpetic eye disease.

\section{Methods}

Mice. Female 4- to 5-week-old BALB/c mice were purchased from Harlan Sprague Dawley Inc. (Indianapolis, Indiana, USA). Female 4- to 5-week-old $129 \mathrm{~Sv} /$ Ev mice were purchased from Taconic Farms (Germantown, New York, USA). MMP-9 knockout mice were kindly provided by Robert M. Senior and J. Michael Shipley (Washington University School of Medicine, St. Louis, Missouri, USA). BALB/c and $129 \mathrm{~Sv} / \mathrm{Ev}$ mice were housed conventionally, and knockout mice were housed 
in sterile microisolator cages in the animal facility. To prevent bacterial infection, all mice received treatment with sulfamethoxazole/trimethoprim (Alphama USPD Inc., Baltimore, Maryland, USA) at the rate of $5 \mathrm{ml} / 200$ $\mathrm{ml}$ of drinking water. All investigations followed guidelines of the committee on the Care of Laboratory Animals Resources, Commission of Life Sciences, National Research Council. The animal facilities of the University of Tennessee are fully accredited by the American Association of Laboratory Animal Care.

Virus. HSV-1 strain RE (kindly provided by Robert Lausch, University of Alabama, Mobile, Alabama, USA), HSV-1 KOS 1.1 (kind gift of David Knipe, Harvard Medical School, Boston, Massachusetts, USA), and the mutant virus n12 (ICP $4^{-/-}$) (gift from David Knipe) were used. HSV-1 KOS and RE viruses were grown in Vero cell monolayers (ATCC no. CCL81, American Type Culture Collection, Rockville, Maryland, USA). Ultraviolet (UV) inactivation of the wildtype virus was carried out for 5 minutes. The mutant virus $\mathrm{n} 12\left(\mathrm{ICP} 4^{--}\right)$was propagated and titrated on a Vero cell line expressing the deficient protein, which is an ICP 4-expressing Vero cell line E-5 (4). Infected Vero cells were harvested, titrated, and stored in aliquots at $-80^{\circ} \mathrm{C}$ until used.

Corneal HSV infection. Corneal infections of all mouse groups were conducted under deep anesthesia induced by the inhalant anesthetic methoxyfurane (Methofane; Pittman Moore, Mondelein, Illinois, USA). The mice were lightly scarified on their corneas with a 27 -gauge needle, and a $2.5-\mu \mathrm{l}$ drop containing $10^{6} \mathrm{PFU}$ of HSV-1 $\mathrm{RE}$ for mice was applied to the eye and gently massaged with the eyelids.

Plasmid DNA preparation. Plasmid DNA encoding murine TIMP-1 was kindly provided by Dylan Edwards (University of East Anglia School of Biological Sciences, Norwich, United Kingdom). TIMP-1 DNA was inserted into the pCDNA3 expression vector (Invitrogen Corp., San Diego, California, USA). The pCDNA3 was used as a negative control against TIMP-1 DNA. The plasmid DNAs were purified by polyethylene glycol precipitation by the method of Sambrook et al. (5) with some modifications. The quality of DNA was measured by electrophoresis on $1 \%$ agarose gel. The protein expression of TIMP-1 DNA was determined by RT-PCR and dot blot analysis after in vitro transfection into Chinese hamster ovary $(\mathrm{CHO})$ cells.

Plasmid DNA administration. One hundred micrograms of plasmid DNA was suspended in $4 \mu \mathrm{l}$ of sterile PBS. Corneas were scarified using a 27-gauge needle in a criss-cross pattern, and the plasmid was administered on days 3 and 6 before virus infection.

Clinical observations. The eyes were examined on different days after infection for the development of clinical lesions by slit-lamp biomicroscopy (Kawa Company, Nagoya, Japan), and the clinical severity of keratitis of individually scored mice was recorded. The scoring system was as follows: 0 , normal cornea; +1 , mild corneal haze; +2 , moderate corneal opacity or scarring;
+3 , severe corneal opacity but iris visible; +4 , opaque cornea and corneal ulcer; +5 , corneal rupture and necrotizing stromal keratitis. The severity of angiogenesis was recorded as described previously (6). According to this system, a grade of 4 for a given quadrant of the circle represents a centripetal growth of $1.5 \mathrm{~mm}$ toward the corneal center. The score of the four quadrants of the eye were then summed to derive the neovessel index (range 0-16) for each eye at a given time point (6).

Gelatin zymography. Zymographic assays for gelatinase were performed as described previously (7). In brief, animals were sacrificed, corneas excised and pooled from six eyes, then homogenized. Protein extracts (20 $\mu \mathrm{g})$ of corneas from mice were subjected to SDS-PAGE on gelatin-containing acrylamide gels ( $8 \%$ acrylamide and $1 \%$ gelatin) under nonreducing conditions. After electrophoresis, gels were washed three times with $2.5 \%$ Triton X-100 for 3 hours to remove SDS. Gels were then rinsed briefly with water followed by incubation overnight at $37^{\circ} \mathrm{C}$ in reaction buffer containing $50 \mathrm{mM}$ Tris, $\mathrm{pH} 7.5,0.15 \mathrm{M} \mathrm{NaCl}, 10 \mathrm{mM} \mathrm{CaCl}_{2}$, and $0.05 \%$ (wt/vol) sodium azide. The gels were stained with $0.5 \%$ (wt/vol) Coomassie brilliant blue R-250. Gelatinolytic activity was detected as a transparent band against a dark blue background. Standards of recombinant human MMP-9 and MMP-2 (Chemicon International, Temecula, California, USA) were included on the gels for identification. Quantitative analysis of activity was conducted using a densitometer.
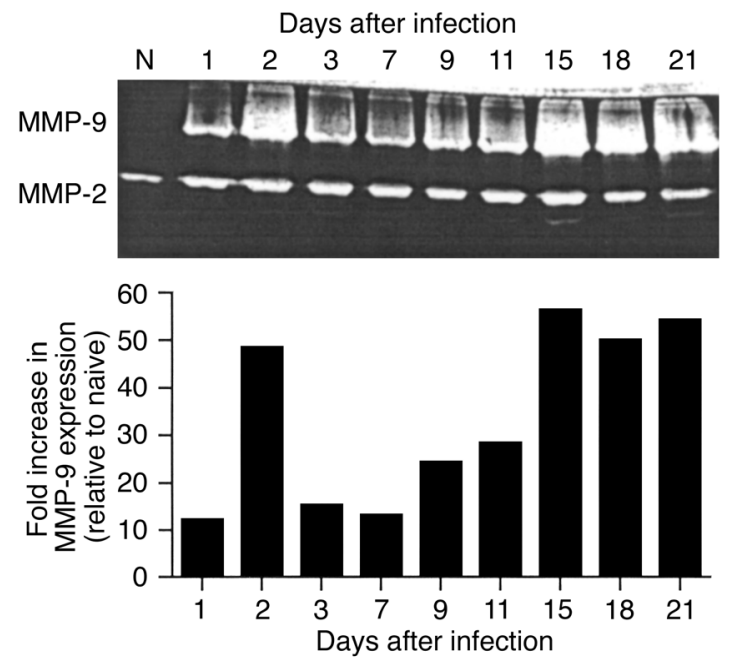

\section{Figure 1}

MMP-9 is present in cornea during HSK, and MMP-9 activities are highly correlated with HSK lesion severity. Groups of animals were infected with $10^{6} \mathrm{PFU}$ of HSV-1 RE on their scarified corneas. The mice were examined clinically by slit-lamp microscopy, and the severity of lesion was scored on a 0 to 5 scale. At day 2 after infection, animals were sacrificed, and corneas were excised and pooled from six eyes and homogenized. The concentrations of protein were measured by the Bradford method. Corneal extract samples ( $20 \mu \mathrm{g} /$ lane) were analyzed by gelatin zymography. Numbers above the gel represent days after infection. High MMP-9 activities were measured at day 2, 15,18 , and 20 after infection. Data represent the results of a single experiment, which was one of three with similar results. 
a
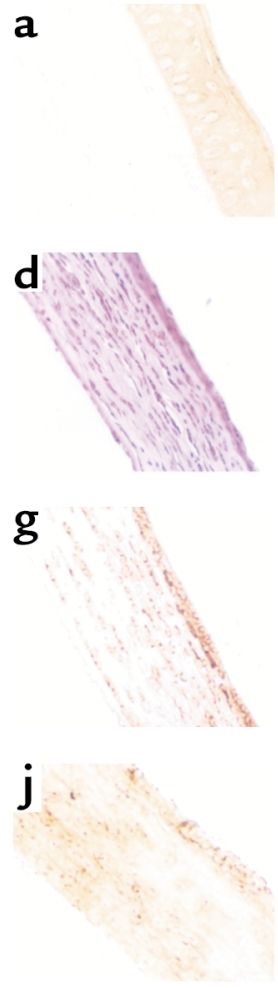

h

b

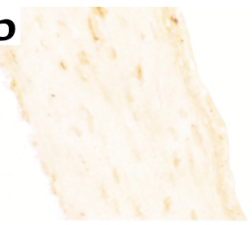

e
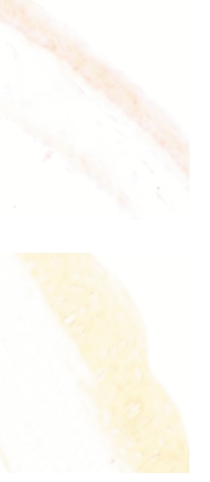

C

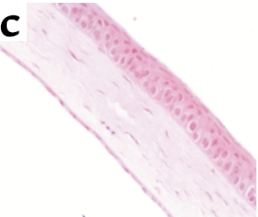

f

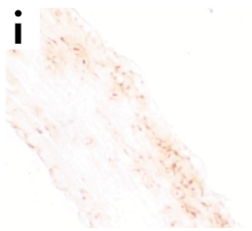

Figure 2

TIMP-1 DNA expression and MMP-9 and neutrophil infiltration into corneas of BALB/c and MMP-9 knockout mice. BALB/c and MMP-9 knockout mice were infected with $10^{6}$ PFU HSV-1 RE or UV-inactivated RE on their scarified corneas. At day 2 after infection, mice were sacrificed, and the eyes were snap-frozen in OCT compound. ( $\mathbf{a}$ and $\mathbf{b}$ ) TIMP-1 expression in (a) naive and (b) TIMP-1-treated groups. (c and $\mathbf{d}$ ) Histopathology of infiltrating cells in the cornea of BALB/c. (c) Naive and (d) RE, day 2 after infection. (e-g) Immunohistochemistry for MMP-9. (e) Naive, (f) UV-inactivated RE day 2 after infection, and (g) RE day 2 after infection. (h-j) Immunohistochemistry for Gr-1. (h) UV-inactivated RE, (i) 129 Sv/Ev, and (j) MMP-9 KO. $\times 200$.

Immunohistochemistry. Eyes were removed and snap-frozen in OCT compound (Miles Inc., Elkhart, Indiana, USA). Sections ( $6 \mu \mathrm{m}$ thick) were cut, air-dried, and fixed in cold acetone for 10 minutes. The sections were then blocked with 3\% BSA and stained with anti-mMMP-9 (Santa Cruz Biotechnology Inc., Santa Cruz, California, USA) for 3 hours, which were followed with biotinylated anti-goat Ig (Santa Cruz Biotechnology Inc.) for 1 hour. Frozen sections were also stained with biotinylated anti-Gr-1

Depletion of neutrophils with $m A b$. Clone RB6-BC5 was kindly provided by E. Balish (University of Wisconsin Medical School, Madison, Wisconsin, USA) with permission of R.L. Coffman (PharMingen, San Diego, California, USA). The cells were grown in RPMI-1640 with $10 \%$ FBS. Hybridoma cells were injected intraperitoneally $\left(5 \times 10^{6}\right.$ cells/mouse $)$ into BALB/c nude mouse. Ascitic fluid was collected, centrifuged at $400 \mathrm{~g}$ for 15 minutes, pooled, and stored at $-20^{\circ} \mathrm{C}$ until ready for use. Delipidized ascitic fluid containing rat IgG-2b Ab's against HLA-DR5 (clone SFR3-DR5; American Type Culture Collection) was used as isotype control. The ascitic fluids were titrated for the $\mathrm{Ab}$ content using an indirect ELISA as described previously (8). BALB/c mice were administered $500 \mu \mathrm{g}$ of anti-Gr-1 Ab intraperitoneally on day 3 before and day 1 after treatment with HSV-1 RE on the cornea. Control mice were treated similarly with rat anti-HLA DR5 Ab.

\section{Figure 3}

Neutrophil depletion markedly diminishes MMP-9 production. Groups of mice received intraperitoneally anti-Gr-1Ab twice as described in Methods, and groups of mice received an irrelevant control isotype-matched rat Ab. Depletion of PMN by Gr-1 Ab was effective, and levels of PMN could be kept at low levels during the period of study (data not shown). Groups of mice were infected on the scarified cornea with $10^{6}$ PFU of HSV- 1 RE. At day 2 after infection, animals were sacrificed, and corneas were excised and pooled from six eyes and homogenized. Corneal extract samples $(20 \mu \mathrm{g} /$ lane $)$ were analyzed by gelatin zymography (a). Lane 1: HSV-1-infected corneas after PMN depletion; Lane 2: HSV-1-infected corneas after injection of isotype control Ig. Data represent the results of a single experiment, which was one of three with similar results.
(PharMingen) for 3 hours. The sections were then treated with horseradish peroxidase-conjugated streptavidin $(1: 1,000$; The Jackson Immunoresearch Laboratories Inc., West Grove, Pennsylvania, USA) and 3,3'-diaminobenzidine substrate (BioGenex Laboratories, San Ramon, California, USA).

Corneal micropocket assay. In vivo angiogenic activity was assayed in the avascular cornea of BALB/c mouse eyes, as described previously (9). Briefly, mice were pretreated with plasmid DNA encoding TIMP-1 twice, intraocularly, before implantation. Pellets for insertion into the cornea were made by combining rhVEGF
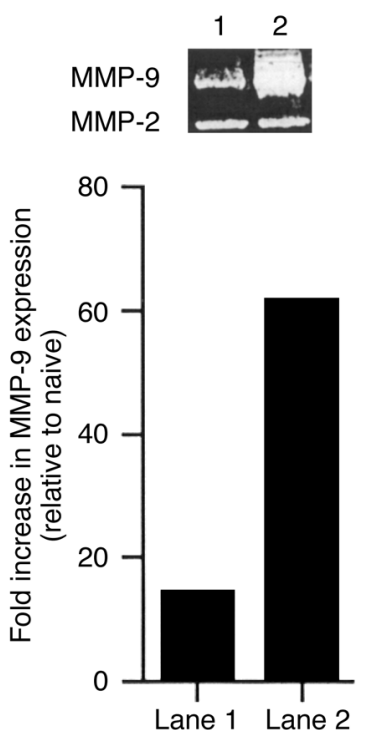


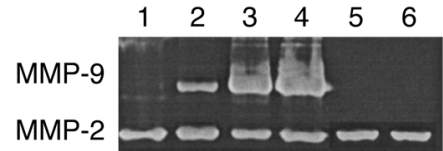

\section{Figure 4}

Replication-defective HSV mutants fail to produce MMP-9. BALB/c mice were infected on the cornea with $10^{6} \mathrm{PFU}$ of the specified virus after scarifying the cornea with a 27-gauge needle. At day 2 after infection, animals were sacrificed, and corneas were excised and pooled from six eyes and homogenized. The concentrations of protein were measured by the Bradford method. Corneal extract samples $(20 \mu \mathrm{g} / \mathrm{lane})$ were analyzed by gelatin zymography. Similar results were obtained in three independent experiments. Lane 1: $10^{6}$ PFU of UV-inactivated HSV-1 RE; lane 2: $10^{6} \mathrm{PFU}$ of ICP $4^{-/-}$; lane 3: $10^{6} \mathrm{PFU}$ of HSV-1 KOS; lane 4: $10^{6} \mathrm{PFU}$ of HSV-1 RE; lane 5: trauma control; lane 6: naive.

(40 $\mu \mathrm{g} ; \mathrm{R} \& \mathrm{D}$ Systems Inc., Minneapolis, Minnesota, USA), sulcralfate (10 mg; BulchMeditec, Vaerlose, Denmark), and hydron polymer in ethanol $(120 \mathrm{mg} / 1 \mathrm{ml}$ ethanol; Interferon Sciences, New Brunswick, New Jersey, USA), and applying the mixture to a $15 \times 15-\mathrm{mm}^{2}$ piece of synthetic mesh (Sefar America Inc., Kansas City, Missouri, USA). The mixture was allowed to air dry, and fibers of the mesh were pulled apart, yielding pellets containing $90 \mathrm{ng}$ VEGF. Pellets containing rhVEGF were implanted into an intracorneal pocket (1 $\mathrm{mm}$ from the limbus). Then the eyes were evaluated for corneal neovascularization. The extent of the neovessel ingrowth was recorded by direct measurement using calipers (Biomedical Research Instruments, Rockville, Maryland, USA) under stereomicroscopy. The number of vessels originating from the limbus was counted over the entire orbit, and the area of angiogenesis was calculated according to the formula for an ellipse: $A=[($ clock hours $) \times 0.4 \times($ vessel length in millimeters) $\times \pi] / 2$. Each clock hour is equal to 30 at the circumstance.

Statistical analysis. Significant differences between groups were evaluated using student's $t$ test.

\section{Results}

$H S V$ infection results in MMP-9 expression. Corneas were taken at various time points after ocular infection with HSV-1 and analyzed by zymography for the presence of the collagen-degrading enzymes MMP-2 and MMP-9. Two bands of activity were evident. A band identified as MMP-2 was present in normal corneal extracts. Moreover, its levels appeared not to vary at different times after virus infection (Figure 1). A second band, identified as MMP-9, was not present in normal tissue, but was present in varying amounts in extracts from virus-infected animals. A peak of activity appeared at 48 hours after infection followed by a decline, then subsequently a second rise of activity starting around day 7. The secondary peak corresponded with the developing clinically evident HSK (Figure 1).

The primary peak of MMP-9 activity was suspected to derive from neutrophils, which were shown previously to be prominent stromal invaders at around 2 days after infection $(10,11)$. Evidence that such invading neutrophils were a source of MMP-9 was obtained by different lines of experimentation. First, histological sections of corneas at 2 days after infection revealed prominent inflammatory cell infiltrates (Figure 2d). The majority of invading cells were neutrophils, most of which could be shown by immunocytochemistry to possess intracellular MMP-9 (Figure 2f). Cells positive for MMP-9 were not found in the virus-infected epithelium, nor was it evident in any cells in sections from uninfected animals. A second approach implicating neutrophils as a source of MMP-9 came from experiments that showed that if neutrophils were deplet-ed from animals before and after infection by treat-ment with specific anti-neutrophil $\mathrm{mAb}$, then levels of MMP-9 were diminished (Figure 3).

Finally, infection of eyes with UV-inactivated virus or replication-defective HSV mutants, which induce negligible neutrophil responses (10), produced either nondetectable (UV-inactivate HSV) or very minor (ICP $4^{-/-}$) levels of MMP-9 production (Figure 4). Taken together, these experiments support a role for corneal stromainvading neutrophils as a principal source of MMP-9 production early after ocular HSV infection.

MMP-9 knockout mice show diminished angiogenesis and reduced severity of HSK. Mice lacking MMP-9 expression because of gene knockout have reduced angiogenesis in several tumor systems $(12,13)$. As shown in Figure 5, a similar outcome was evident in mice ocularly infected with HSV-1. Thus, in a comparison of the extent of ocular angiogenesis in $M M P_{-1 /-}$ mice compared with 125 Sv/Ev control animals, levels were around $50 \%$ less in the $M M P-9^{-/-}$animals when measured over a 20 -day test period. These differences were shown to be statistically significant $(P<0.01)$. Furthermore, when animals were

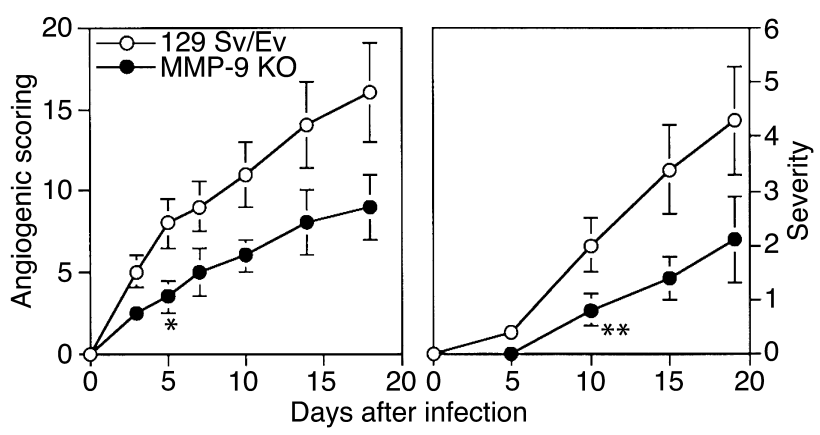

\section{Figure 5}

MMP-9 knockout mice are protected from HSK pathogenesis. Groups of 129 Sv/Ev wild-type (4-5 weeks old) and MMP-9 knockout mice (4-5 weeks old) were infected with $10^{6}$ PFU of HSV-1 RE on their scarified corneas. The mice were examined clinically by slit-lamp microscopy, and the severity of lesions was scored on a 0 to 5 scale. The mean clinical scores at day 5,10,15, and 19 are plotted for all groups. Also, the animals were examined for the extent of angiogenesis as described in Methods. The data are compiled from three independent experiments with seven individual mice in each group. $P \leq 0.05$ between wild-type and knockout mice at day 5 and beyond (angiogenic scoring; asterisk) and at day 10 and beyond (HSK lesion severity; double asterisk). 


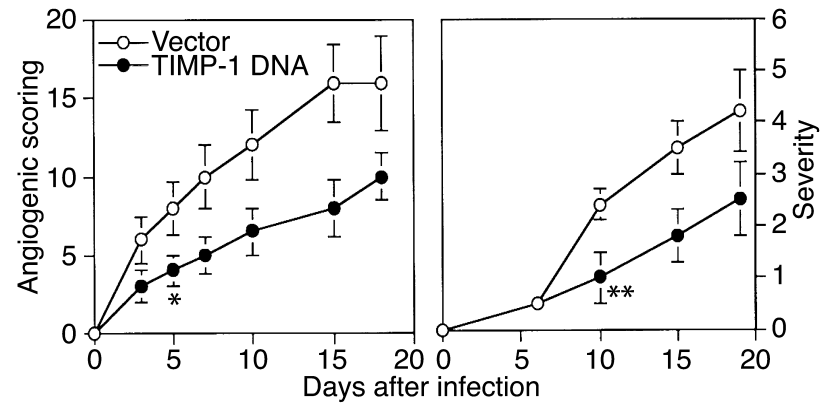

Figure 6

TIMP-1 DNA inhibits angiogenic scoring and HSK lesion severity. Groups of animals were treated twice with $100 \mu \mathrm{g}$ of TIMP-1 DNA and vector DNA intraocularly 6 and 3 days before virus infection and were infected with $10^{6} \mathrm{PFU}$ of HSV-1 RE on their scarified corneas 3 days after a second treatment of DNAs. The mice were examined clinically by slit-lamp microscopy, and the severity of lesions and the extent of angiogenesis were scored as described in Methods. Data are compiled from three independent experiments with seven individual mice per group. Significant differences $(P \leq 0.05)$ between TIMP-1 DNA- and vector DNA-treated mice are indicated by an asterisk (angiogenic scoring) and a double asterisk (severity). Such differences were present at day 5 and beyond (angiogenic scoring) and at day 10 and beyond (HSK lesion severity).

followed to measure the severity of HSK on days 12 and 21 after infection, the severity was significantly less in the MMP-9-- animals. In an additional experiment, ocularly infected $M M P_{-} 9^{-/-}$animals were analyzed to determine if a stromal neutrophil response was evident in such animals. As shown in Figure 2c, a prominent response was indeed present at 48 hours after infection.

Effect of MMP-9 inbibitor on HSV-induced angiogenesis. The MMP-9 is known to be inhibited by TIMP-1, with this molecule serving to minimize the duration of MMP-9 activity in some tumor angiogenesis systems (14). To measure the effect of TIMP-1 on HSV-1-induced MMP-9 induction, animals were given either plasmid DNA encoding TIMP-1 or control plasmid DNA vector on the cornea. Animals were treated at 3 and 6 days prior to HSV-1 infection, after which the extent of angiogenesis was compared in TIMP-1 and vector DNA-treated animals. Preliminary experiments in uninfected mice revealed that TIMP-1 DNA treatment led to TIMP-1 expression in frozen eye sections (Figure $2 \mathrm{~b})$. The results in Figure 6 indicate that the extent of angiogenesis in TIMP-1 plasmid DNA-treated animals was significantly decreased compared with vector-treated animals during the 3- to 5-day observation period. As a further measure of the effect of TIMP- 1 inhibition on MMP-9, corneal extracts were taken at 48 hours from TIMP-1 and vector DNA-treated animals for measurement of MMP-9 levels by zymography. The results shown in Figure 7 demonstrate significantly diminished MMP-9 signals in samples from TIMP-1-treated animals. These experiments provide further evidence for a role of MMP-9 in HSV-induced angiogenesis.

TIMP-1 DNA inbibits VEGF-induced angiogenesis by its effect on MMP-9 activity. In a previous report, we demonstrated that ocular infection with HSV induces the potent angiogenesis factor VEGF family of proteins, with these playing an important role in corneal angiogenesis (2). Since TIMP-1 is not expected to inhibit VEGF and these molecules are present following HSV infection, observing the inhibitory effects of TIMP-DNA on HSV angiogenesis was perhaps surprising. Hence, experiments were done using a corneal micropocket assay to measure the effect of pretreating eyes with TIMP-1 DNA on the angiogenic response to VEGF. The results shown in Figure 8 indicate that such pretreatment diminished neovascularization to about $50 \%$ of VEGF control. To determine if levels of MMP-9 might account for such differences, corneal extracts from mice with control and VEGF-containing micropockets were tested by zymography for MMP-9. Unlike normal corneas, VEGFimplanted eyes do produce MMP-9. Moreover, in TIMP-1 DNA-pretreated VEGF-implanted eyes MMP-9 levels were markedly diminished compared with VEGF-implanted vector-treated eyes (Figure 9). These results demonstrate that MMP-9 represents a significant component of HSV-induced angiogenesis being also involved indirectly in angiogenesis caused by other known angiogenesis factors.

\section{Discussion}

In this report, we demonstrate that HSV infection of the cornea results in the upregulation of the matrix-degrading metalloproteinase enzyme MMP-9. This enzyme
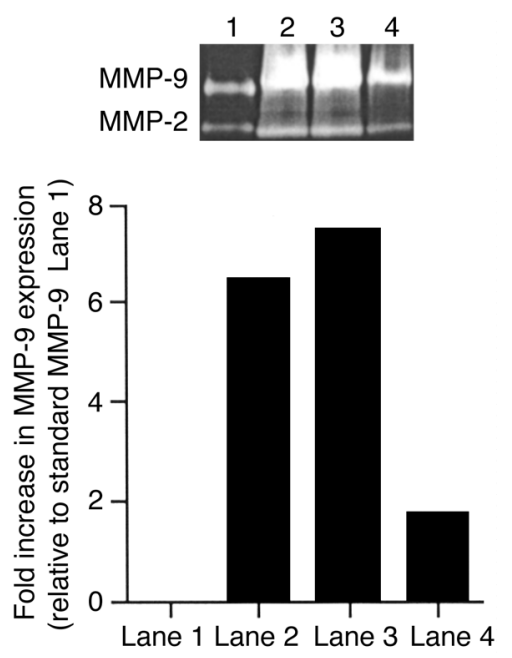

\section{Figure 7}

TIMP-1 DNA inhibits MMP-9 activity at day 2 after infection. Groups of animals were treated twice with $100 \mu \mathrm{g}$ of TIMP-1, vector, and $\beta$-gal DNA intraocularly 6 and 3 days before virus infection and were infected with $10^{6} \mathrm{PFU}$ of HSV-1 RE on their scarified corneas 3 days after a second treatment of DNAs. At day 2 after infection, animals were sacrificed, and corneas were excised and pooled from six eyes and homogenized. Corneal extract samples (20 $\mu \mathrm{g} /$ lane) were analyzed by gelatin zymography. Lane 1: human zymography standard; lane 2: corneas treated with vector DNA; lane 3: corneas treated with $\beta$-gal DNA; lane 4: corneas treated with TIMP-1 DNA. The data represent a single experiment from two independent experiments with four mice in each group. 


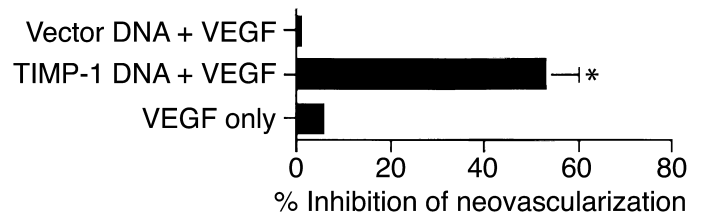

\section{Figure 8}

Pretreatment of TIMP-1 DNA inhibits recombinant VEGF-induced angiogenesis. Groups of mice were treated intraocularly with TIMP-1 DNA or vector DNA, and hydron pellets containing rhVEGF (90 ng) were implanted into the corneal pockets. The total number of neovessels originating in the limbus and the area of neovascularization were calculated. Data are compiled from two separate experiments (five mice per group) with similar results. $P<0.05$.

was shown to contribute to the neovascularization process that occurs in the corneal stroma in response to HSV infection. The likely source of MMP-9, at least initially after infection, was neutrophils that are signaled to invade the cornea soon after infection. Corneal-infiltrating neutrophils were shown to express MMP-9, and preventing the neutrophil response with specific $\mathrm{mAb}$ diminished MMP-9 expression as well as the extent of angiogenesis. Further supporting a role for MMP-9 in HSV-induced corneal angiogenesis was the observation that inhibition of MMP-9 with the specific inhibitor TIMP-1 resulted in reduced angiogenesis. In addition, angiogenesis was diminished in ocularly infected $M M P^{-9^{-/}}$mice. Our results demonstrate that MMP-9 is involved in angiogenesis caused by HSV. Since angiogenesis appears to represent a vital step in the pathogenesis of herpetic stromal keratitis (15), these results indicate that targeting MMP-9 for inhibition should prove useful for the therapy of HSK.

Neovascularization of the cornea is a prominent event following HSV infection of the mouse eye. Growth of new blood vessels from the limbus are evident as early as 24 hours after infection, with vessels in some instances growing to the central cornea by 14 days after infection (16). By this time, lesions of HSK have become evident. Characteristically, the virus replicates for only a few days in corneal epithelial cells, but angiogenesis and the stromal immunoinflammatory reaction progress in severity for $2-3$ weeks $(17,18)$. Since inhibiting angiogenesis, at least if begun early, diminished the severity of HSK, we have suggested that neovascularization of the cornea is a necessary step in the pathogenesis of HSK (18). It likely serves to provide access to the cornea of $T$ lymphocytes that orchestrate the immunoinflammatory lesions of HSK. A crucial unsolved problem is to explain how a relatively brief period of HSV replication in the corneal epithelium leads to the rapid angiogenic sprouting from vessels at the corneal limbus, which ultimately may involve almost the whole cornea. We anticipate that the infection signals the production of many molecules involved both in angiogenesis and inflammation. This report is the first, to our knowledge to document a potential role of MMP-9 in HSV-induced angiogenesis. However, MMP-9 is well-known to be involved in several tumor angiogenesis systems $(19,20$, 21 ) and has also been shown to participate in ocular wound healing $(22,23)$.

The role MMP-9 is assumed to play in angiogenesis is that of to facilitating the growth of new blood vessels by breaking down the extracellular matrix (24). As observed in our experiments, the lack of MMP-9 reduced but did not abrogate angiogenesis. This result was not unexpected, since many additional groups of molecules can participate in angiogenesis, usually by distinct stimulatory effects on vascular endothelial cells $(25,26)$. For instance, we know from previous results with ocular HSV infection that the VEGF family of proteins are involved in angiogenesis (2). In addition, certain chemokines produced following HSV-1 ocular infection, such as MIP-2 (27), might also contribute to angiogenesis. Given this complexity, it may prove difficult in clinical situations to successfully control angiogenesis unless a cocktail of inhibitors are employed.

MMP-9, however, represents a logical target for therapy, since this component acts as the principal degrader of the extracellular matrix (ECM), at least in certain tumor angiogenesis systems $(28,29)$. Moreover, ECM degradation appears to represent an essential step in angiogenesis (30), facilitating the growth of angiokinestimulated vascular components. Consequently, the function of MMP-9 is expected to amplify the effect of other angiogenesis factors. Indeed, in our studies we demonstrated that inhibiting MMP-9, as could be done with the specific inhibitor TIMP-1, also served to inhibit angiogenesis mediated by VEGF. Such results could mean that the combined use of reagents that inhibit MMP-9, along with specific inhibitors of certain angiogenesis factors, may achieve more complete control of ocular angiogenesis. We are currently testing such notions in our HSV angiogenesis model.

Early after HSV infection, the cellular source of MMP-9 was shown to be the neutrophils that promptly invade the corneal stroma following HSV infection of the overlying epithelium. Preventing this, neutrophil response both diminished detectable

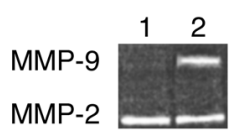

\section{Figure 9}

TIMP-1 DNA reduces VEGF-induced angiogenesis by inhibition of MMP-9 activity. Groups of mice were treated intraocularly with TIMP-1 DNA or vector DNA, and hydron pellets containing rh VEGF $(90 \mathrm{ng}$ ) were implanted into the corneal pellets. At day 4 after treatment, animals were sacrificed, and corneas were excised and pooled from eight eyes and homogenized. The concentrations of protein were measured by the Bradford method. Corneal extract samples (20 $\mu \mathrm{g} /$ lane) were analyzed by gelatin zymography. Similar results were obtained in three independent experiments. Lane 1: corneal sample from TIMP-1 DNA-treated group; lane 2: corneal sample from vector DNA-treated group. 
levels of MMP-9 and reduced the extent of angiogenesis. However, it is not clear if neutrophils remain as the only source of MMP-9 or, in fact, if the function of MMP-9 is necessary after the initial stages of HSVinduced angiogenesis. It could well be that only a certain degree of corneal neovascularization is actually required to permit invasion by inflammatory $\mathrm{T}$ cells and other cell types involved in lesions of HSK. In line with this, our preliminary investigations have indicated that there is no benefit, in terms of the progress of HSK, of inhibiting neovascularization after the initial stage. Ultimately, to fully control HSK lesions could require approaches that reverse established angiogenesis. This issue is under investigation in our laboratory.

\section{Acknowledgments}

This work is supported by NIH grant EY-05093. We are grateful to Tommy Jordan for his computer technical assistance.

1. Kumaraguru, U., Davis, I., and Rouse, B.T. 1999. Chemokines and ocular pathology caused by corneal infection with herpes simplex virus. J. Neurovirol. 5:42-47.

2. Zheng, M., Deshpande, S., Lee, S., Ferrara, N., and Rouse, B.T. 2001. Contribution of vascular endothelial growth factor in the neovascularization process during the pathogenesis of herpetic stromal keratitis. J. Virol. 75:9828-9835.

3. Mollinedo, F., Nakajima, M., Llorens, A., Barbosa, E., and Fabra, A. 1997. Major co-localization of the extracellular matrix degradative enzymes heparanase and gelatinase in tertiary granules of human neutrophils. Biochem. J. 327:917-923.

4. Sheppard, A.A., and De Luca, N.A. 1989. Intragenic complementation among partial peptides of herpes simplex virus regulatory protein ICP 4. J. Virol. 63:1203-1211.

5. Sambrook, J., Fritsch, E.F., and Maniatis, T. 1989. Molecular cloning: a lab oratory manual, 2 nd ed. Cold Spring Harbor Laboratory Press. Plainview, New York, USA.

6. Dana, M.R., Zhu, S., and Yamada, J. 1998. Topical modulation of interleukin-1 activity in corneal neovascularization. Cornea. 17:403-409.

7. Twining, S.S., et al. 1996. Effect of vitamin A deficiency on the early response to experimental Pseudomonas Keratitis. Invest. Ophthalmol. Vis. Sci. 37:511-522.

8. Doymez, M.Z., and Rouse, B.T. 1992. Herpetic stromal keratitis: an immunopathologic disease and mediated by $\mathrm{CD} 4{ }^{+} \mathrm{T}$ lymphocytes. Invest. Ophthalmol. Vis. Sci. 33:2165-2173.
9. Kenyon, B.M., et al. 1996. A model of angiogenesis in the mouse cornea. Invest. Ophthalmol. Vis. Sci. 37:1625-1632.

10. Thomas, J., Gangappa, S., Kanangat, S., and Rouse, B.T. 1997. On the essential involvement of neutrophils in the immunopathologic disease. J. Immunol. 158:1383-1391.

11. Tumpey, T.M., Chen, S.H., Oakes, J.E., and Lausch, R.N. 1996. Neutrophil mediated suppression of virus replication after herpes simplex virus type I infection of the murine cornea. J. Virol. 70:898-904.

12. Vu, T.H., et al. 1998. MMP-9/gelatinase B is a key regulator of growth plate angiogenesis and apoptosis of hypertropic chondrocytes. Cell. 93:411-422.

13. Itoh, T., et al. 1999. Experimental metastasis is suppressed in MMP-9deficient mice. Clin. Exp. Metastasis. 17:177-181.

14. Rigg, A.S., and Lemoine, N.R. 2001. Adenoviral delivery of TIMP1 or TIMP2 can modify the invasive behavior of pancreatic cancer and can have a significant antitumor effect in vivo. Cancer Gene Ther. 8:869-878.

15. Lee, S., et al. 2002. IL-12 suppresses the expression of ocular immunoinflammatory lesions by effects on angiogenesis. J. Leukoc. Biol. 71:467-476.

16. Deshpande, S.P., Zheng, M., Lee, S., and Rouse, B.T. 2002. Mechanisms of pathogenesis in herpetic immunoinflammatory ocular lesions. Vet. Microbiol. 86:17-26.

17. Thomas, J., Gangappa, S., Chen, S., Daheshia, M., and Rouse, B.T. 1998. Immunopathogenesis of hepetic stromal keratitis-a blinding disease of mankind. Central European Journal of Immunology. 23:72-83.

18. Zheng, M., Schwarz, M.A., Lee, S., Kumaraguru, U., and Rouse, B.T. 2001. Control of stromal keratitis by inhibition of neovascularization. Am. J. Pathol. 159:1021-1029.

19. Sang, Q.X. 1998. Complex role of matrix metalloproteinase in angiogenesis. Cell. Res. 8:171-177.

20. Bergers, G., et al. 2000. Matrix metalloproteinase- 9 triggers the angiogenic switch during carcinogenesis. Nat. Cell. Biol. 2:737-744.

21. Himelstein, B.P., Canete-Soler, R., Bernhard, E.J., Dilks, D.W., and Muschel, R.J. 1995. Metalloproteinases in tumor progression: the contribution of MMP-9. Invasion Metastasis. 14:246-258.

22. Li, D.Q., et al. 2001. Regulation of MMP-9 production by human corneal epithelial cells. Exp. Eye Res. 73:449-459.

23. Clark, A.F. 1998. New discoveries on the roles of matrix metalloproteinases in ocular cell biology and pathology. Invest. Ophtalmol. Vis. Sci. 39:2514-2516.

24. Moses, M.A. 1997. The regulation of neovascularization by matrix metalloproteinases and their inhibitors. Stem Cell. 15:180-189.

25. Yankopoulos, G.D., et al. 2000. Vascular-specific growth factor and blood vessel formation. Nature. 407:242-248.

26. Carmeliet, P., and Rakesh, K.J. 2000. Angiogenesis in cancer and other disease. Nature. 407:249-257.

27. Yan, X.T., Tumpey, T.M., Kunkel, S.L., Oakes, J.E., and Lausch, R.N. 1998. Role of MIP-2 in neutrophil migration and tissue injury in the herpes simplex virus-1-infected cornea. Invest. Ophthalmol. Vis. Sci. 39:1854-1862.

28. Opdenakker, G., et al. 2001. Gelatinase B functions as regulator and effector in leukocyte biology. J. Leukoc. Biol. 69:851-859.

29. Mignatti, P., and Rifkin, D.B. 1996. Plasminogen activators and matrix metalloproteinases in angiogenesis. Enzyme Protein. 49:117-137.

30. Liotta, L.A., Steeg, P.S., and Stetler-Stevenson, W. 1991. Cancer metastasis and angiogenesis: an imbalance of positive and negative regulation. Cell. 64:327-336. 